\title{
Interactions and Optimizations Analysis between Stiffness and Workspace of 3-UPU Robotic Mechanism
}

\author{
Dan Zhang, Bin Wei \\ Department of Mechanical Engineering, Lassonde School of Engineering, York University, 4700 Keele Street, Toronto ON. \\ M3J1P3Canada,dzhang99@yorku.ca,binwei28@yorku.ca
}

\begin{abstract}
The interactions between stiffness and workspace performances are studied. The stiffness in $\mathrm{x}, \mathrm{y}$ and $\mathrm{z}$ directions as well as the workspace of a 3-UPU mechanism are studied and optimized. The stiffness of the robotic system in every single moveable direction is measured and analyzed, and it is observed that in the case where one tries to make the $\mathrm{x}$ and $\mathrm{y}$ translational stiffness larger, the $\mathrm{z}$ directional stiffness will be reduced, i.e. the $\mathrm{x}$ and $\mathrm{y}$ translational stiffness contradicts with the one in $\mathrm{z}$ direction. Subsequently, the objective functions for the summation of the $\mathrm{x}$ and $\mathrm{y}$ translational stiffness and $\mathrm{z}$ directional stiffness are established and they are being optimized simultaneously. However, we later found that these two objectives are not in the same scale; a normalization of the objectives is thus taken into consideration. Meanwhile, the robotic system's workspace is studied and optimized. Through comparing the stiffness landscape and the workspace volume landscape, it is also observed that the $\mathrm{z}$ translational stiffness shows the same changing tendency with the workspace volume's changing tendency while the $\mathrm{x}$ and $\mathrm{y}$ translational stiffness shows the opposite changing tendency compared to the workspace volume's. Via employing the Pareto front theory and differential evolution, the summation of the $\mathrm{x}$ and $\mathrm{y}$ translational stiffness and the volume of the workspace are being simultaneously optimized. Finally, the mechanism is employed to synthesize an exercise-walking machine for stroke patients.
\end{abstract}

Keywords: Robotic manipulator, stiffness, workspace, interactions, optimization, walking machine.

\section{INTRODUCTION}

Parallel mechanisms have been utilized in different fields such as conduct manufacturing machining [1]-[3], medical devices [4]-[7], sensor applications [8], etc., contributing to parallel mechanisms' parallel structure arrangement (i.e. high rigidity, high accuracy, high speed and acceleration, and no cumulative joint/link error). Stiffness and workspace analysis are widely addressed topics. There are numerous resources dealing with stiffness and workspace of parallel mechanisms [9]-[13]. Due to the space limitation, here the authors just list some typical examples of them. Stiffness can be one of the most critical factors to be considered as high stiffness can result in better precision. For example, in machine tools applications, if the machine tools have poor stiffness performance, the machined piece will be very rough. The main diagonal items of the stiffness matrix are the stiffness in each corresponding direction and they can represent the pure stiffness more clearly, this has been proved by the FEA examination [14], and these items can be the primary components when one analyzes and optimizes the stiffness. In [15], the average value and standard deviation of the compliance matrix's main diagonal items for a parallel mechanism were employed to describe and optimize the kinetostatic performance. However, the authors did not consider the unit incompatibility of each element of the compliance matrix when determining the average value and the standard deviation of the compliance matrix's main diagonal items. In [16], the main diagonal items of the stiffness matrix were utilized as an objective function to analyze a parallel manipulator's stiffness; this study followed the study in [15]. In [17], the stiffness in $\mathrm{z}$ direction was employed as a factor to represent the entire machine's overall stiffness. In [18], a general performance index was used for optimizing the stiffness of a robotic manipulator. The drawback of parallel manipulators, compared to their serial ones, is that parallel mechanisms reach smaller workspace. For a parallel manipulator, its workspace is the space to which the moving platform's center is able to reach. One usually desires a large workspace for parallel mechanisms, for example, having a large workspace capacity for parallel mechanisms used in machine tools usually indicates that the machine tool-head is able to stretch out to every corner of the work-piece when one is running milling, cutting, deburring, drilling, and finishing operations. In [19], a condition index was put forward as a criterion for optimizing the workspace for a three degree-of-freedom robotic mechanism for achieving a "well-conditioned" workspace performance. In [20] and [21], the volume of the workspace of robotic mechanisms was employed as a criterion for optimizing the workspace. 
For the majority of cases, the workspace volume of robotic mechanisms is usually employed as a criterion for assessing and optimizing the workspace performance.

Through analyzing, it is observed that the parallel mechanisms' stiffness can get affected during the time one optimizes the workspace. Usually both the stiffness and workspace performances need to be taken into consideration in some applications; hence, performing a multi-objective optimization on these two terms is necessary. In this study, the stiffness and workspace are single optimized and analyzed firstly; subsequently these two terms are optimized and analyzed simultaneously.

Numerous optimization approaches can be employed, for example, differential evolution, particle swarm optimization, ant colony optimization, and genetic algorithm. In [14], an approach that is based on the particle swarm optimization was employed for optimizing the dexterous stiffness and reachable workspace of the 4UPS-PU robotic mechanism simultaneously. In [22], the authors optimized the dexterity and the workspace of a tripod machine structure at the same time by resorting to the combination of the Pareto frontier sets and evolutionary algorithm. In [23], a multi-objective optimization problem was transformed to a single-objective optimization issue. The average vertical stiffness, volume of the workspace and average dexterity for a hybrid robotic mechanism that is reconfigurable were optimized simultaneously via employing the sequential simplex approach. In [24], the GA and Pareto front set approach were individually utilized for optimizing a tripod based parallel manipulator's stiffness and its workspace.

The differential evolution algorithm usually performs better than particle swarm optimization in the aspects of solution accuracy, robustness and time cost. In most cases, differential evolution is easier to be implemented as compared to other algorithms. In addition, differential evolution is much more efficient and robust in terms of convergent ability and producing the same results in multiple runs as compared to other evolution algorithms; the above conclusion was proved in [25]. Interestingly, to the best of the authors' knowledge, differential evolution algorithm is not often utilized in the area of optimization for parallel mechanisms; meanwhile the normalization of stiffness in every moveable direction was not taken into consideration in most cases. Here with respect to the singleobjective optimization problem, the authors employ the differential evolution algorithm for optimizing the stiffness and workspace of the 3-UPU parallel mechanism. With respect to the multi-objective optimization issue, the summation of the $\mathrm{x}$ and $\mathrm{y}$ translational stiffness and the volume of the robotic mechanism's workspace are being optimized simultaneously through respectively employing the Pareto front theory and differential evolution algorithm.

The organization of this paper is as follows: Section 2 presents the Jacobian matrix analysis of the 3-UPU parallel mechanism; the stiffness analysis of the mechanism and single-objective optimization and some issues are studied in Section 3; Section 4 analyzes the workspace of the mechanism and single-objective optimization and some issues are also discussed the multi-objective optimization for stiffness and workspace is discussed and analyzed in Section 5, and the motivation for choosing the objective functions, the used optimization algorithm and the computational costs are presented as well; Section 6 presents a potential application of the mechanism used in the medical field finally conclusion is given in Section 7.

\section{JACOBIAN OF THE MECHANISM}

Determination of the robotic mechanism's Jacobian matrix is essential for the following analysis. The 3-UPU robotic mechanism possesses three translational degrees of freedom (DOF). Three identical limbs connect the moving platform and the base, as illustrated in Fig.1. For the purpose of analysis, two frames $\mathrm{O}(\mathrm{X}, \mathrm{Y}, \mathrm{Z})$ and $\mathrm{P}(\mathrm{x}, \mathrm{y}, \mathrm{z})$ are attached to the base $\mathrm{B}_{1} \mathrm{~B}_{2} \mathrm{~B}_{3}$ and moving platform $\mathrm{P}_{1} \mathrm{P}_{2} \mathrm{P}_{3}$ center, respectively. Angle $\alpha_{b}$ is from $\mathrm{X}$ axis to $\mathrm{OB}_{1}$; angle $\alpha_{p}$ is from $\mathrm{x}$ axis to $\mathrm{OP}_{1}$.

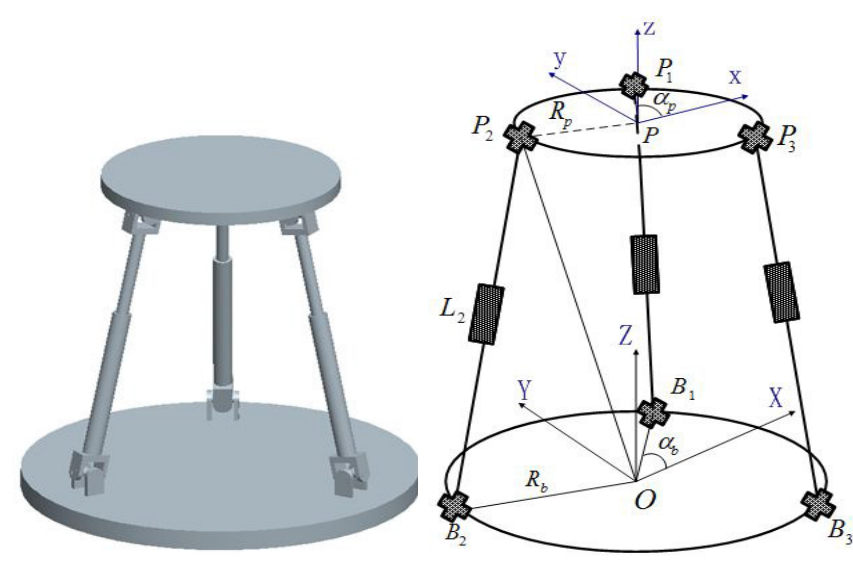

Fig.1. Schematic representation of 3-UPU manipulator and its model.

The velocity of $\mathrm{P}_{\mathrm{i}}$ can be written as:

$$
v_{P i}=\omega_{i} \times s_{i}+\dot{L}_{i} s_{i}
$$

where $s_{i}$ represents the unit vector along the i-th leg, $w_{\mathrm{i}}$ represents the angular velocity of the $i$-th leg relative to the base, and $L i$ represents the length of each leg that is able to be determined via the vector loop equation. Through multiplying $s_{i}$ in both sides of formulation (1) results (2):

$$
J v_{P}=\dot{q}
$$

where $v_{p}$ represents the moving platform center's velocity. The Jacobian matrix of the 3-UPU robotic mechanism is thus determined:

$$
J=\left[\begin{array}{lll}
s_{1}^{T} & s_{2}^{T} & s_{3}^{T}
\end{array}\right]^{T}
$$




\section{STIFFNESS OF THE MECHANISM AND SINGLE-OBJECTIVE OPTIMIZATION}

The stiffness can be considered as a "bridge" between the displacement of the moving platform and a force acting on it, and the 3-UPU robotic mechanism's stiffness at a given spot inside the workspace can be portrayed by a stiffness matrix [26]. The stiffness matrix is determined through employing the kinematic and static formulations:

$$
K=J^{T} K_{J} J=k J^{T} J
$$

The main diagonal items of the stiffness matrix are each corresponding direction's stiffness. As a case study, when $\mathrm{R}_{\mathrm{b}}=3 \mathrm{~cm}, \mathrm{R}_{\mathrm{p}}=1 \mathrm{~cm}, \mathrm{x}=0, \mathrm{y}=0, \mathrm{z}=5 \mathrm{~cm}$, the changing trends of the $\mathrm{x}, \mathrm{y}$ and $\mathrm{z}$ translational stiffness with respect to $\alpha_{b}(\mathrm{rad})$ and $\alpha_{p}(\mathrm{rad})$ are illustrated in Fig.2.
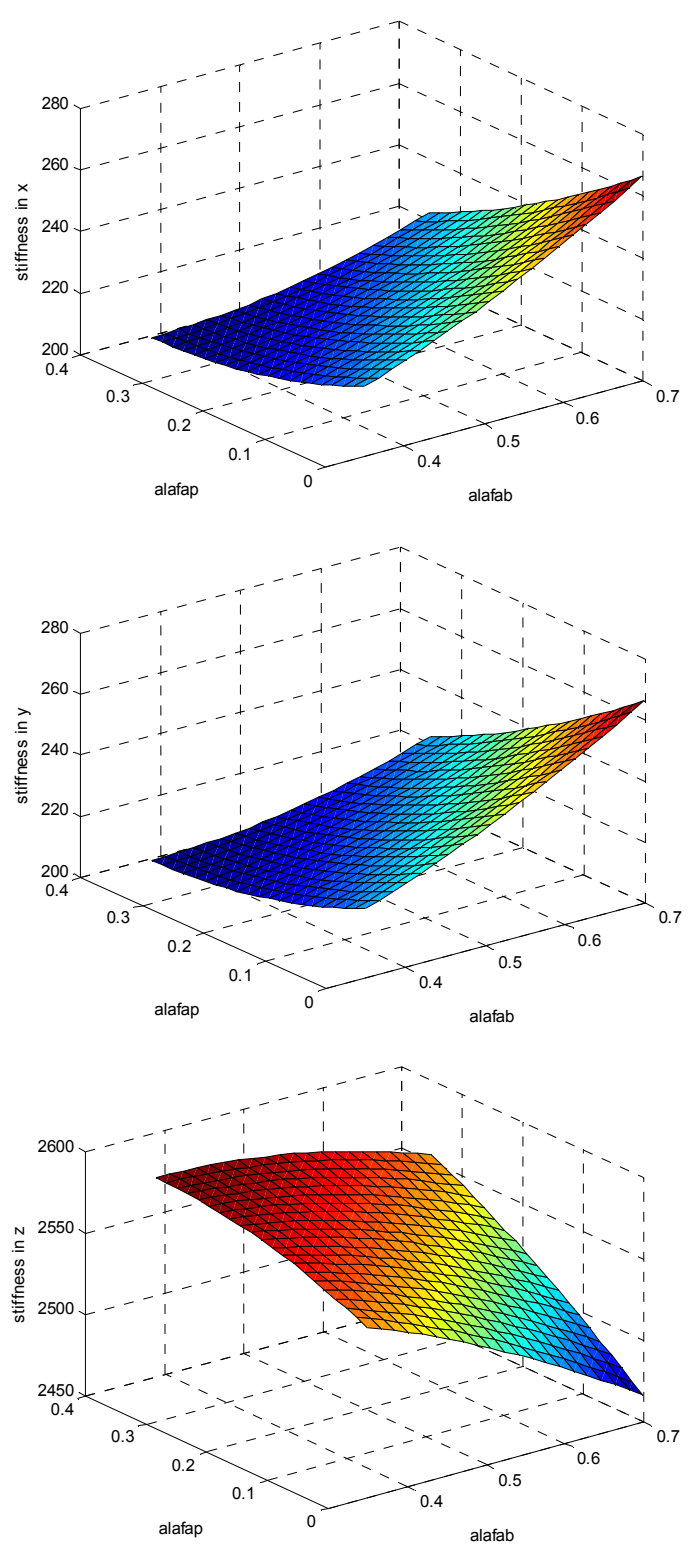

Fig.2. Stiffness in three directions when $R_{b}=3, R_{p}=1$.
In the case where $\mathrm{x}=0, \mathrm{y}=0, \mathrm{z}=5 \mathrm{~cm}, \alpha_{b}=0.5 \mathrm{rad}$, $\alpha_{p}=0.25 \mathrm{rad}$, the changing trends of the $\mathrm{x}, \mathrm{y}$ and $\mathrm{z}$ translational stiffness with respect to $R_{b}$ and $R_{p}$ are demonstrated in Fig.3.
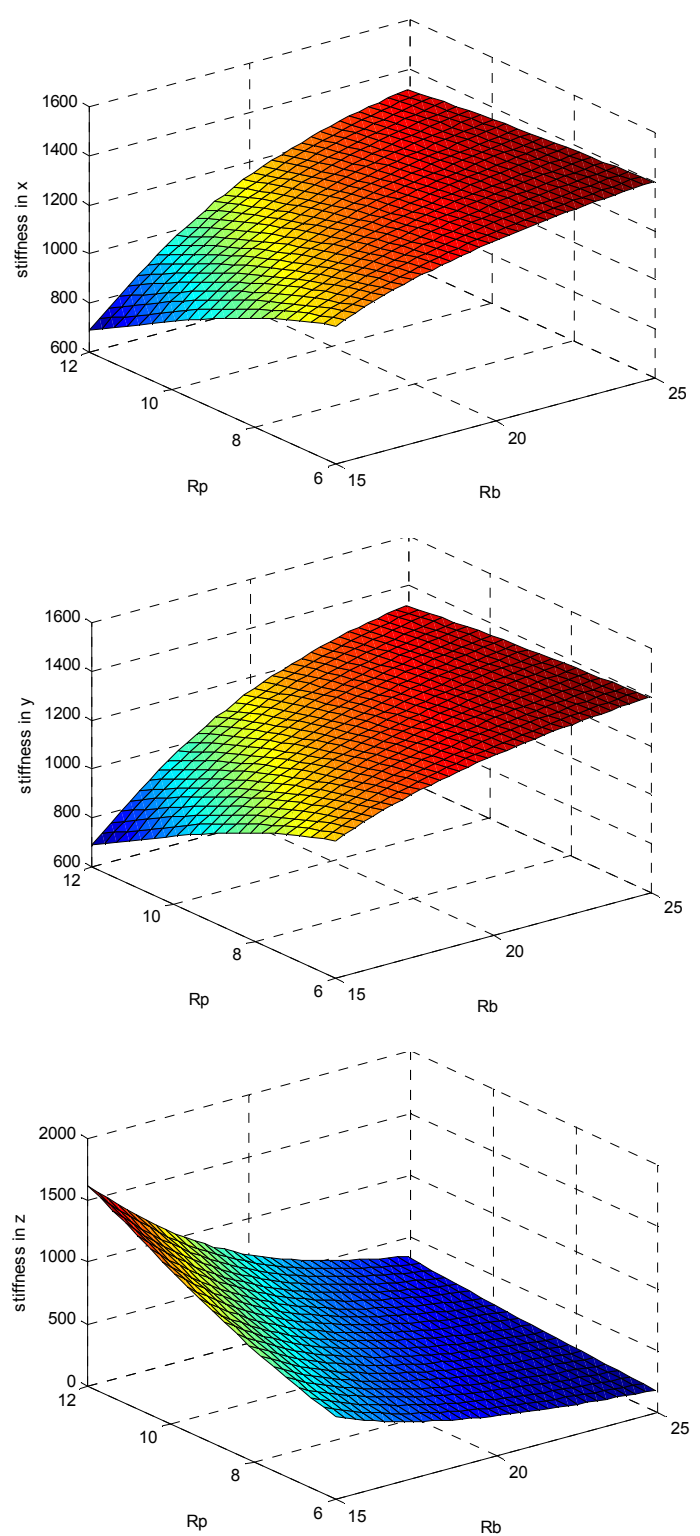

Fig.3. Stiffness in $\mathrm{x}, \mathrm{y}$ and $\mathrm{z}$ direction when

$$
\alpha_{b}=30^{\circ}, \alpha_{p}=15^{\circ} \text {. }
$$

It can be observed that the $\mathrm{x}$ and $\mathrm{y}$ translational stiffness present the same changing fashion while the $\mathrm{z}$ translational stiffness presents the reverse changing fashion as compared to the $\mathrm{x}$ and $\mathrm{y}$ stiffness case. This indicates that in the case where the $\mathrm{x}$ and $\mathrm{y}$ translational stiffness increase, the $\mathrm{z}$ translational stiffness decreases, i.e. these two types of stiffness conflict with one another.

The weighted aggregation function as illustrated below will be employed for optimizing the $\mathrm{x}, \mathrm{y}$ and $\mathrm{z}$ translational stiffness. It transforms a multi-objective optimization issue to a single-objective optimization case through integrating 
multiple objective functions into a single objective function [27]. However, in the case where the objectives are not in the same scale, a normalization of the different objectives needs to be taken into consideration. The formulation shown below is able to be determined via joining two objectives to one:

$$
\begin{array}{r}
O b j F=\lambda_{1} \frac{O b j F 1-O b j F 1_{\text {min }}}{O b j F 1_{\text {max }}-O b j F 1_{\text {min }}} \\
+\lambda_{2} \frac{O b j F 2_{2}-O b j F 2_{\text {min }}}{O b j F 2_{\max }-O b j F 2_{\text {min }}} \\
O b j F 1=K(1,1)+K(2,2) \\
O b j F 2=K(3,3)
\end{array}
$$

where $\mathrm{ObjF}$ represents the objective function, $O b j F 1$ represents the summation of $\mathrm{x}$ and $\mathrm{y}$ directional stiffness, $O b j F 2$ represents the $\mathrm{z}$ directional stiffness, $O b j F i_{\max }$ represents the top limit of the corresponding objectives, $O b j F i_{\text {min }}$ represents the low limit of the objectives, and $\lambda_{i}$ represents the weight.

The design variables are as follows: $\mathrm{R}_{\mathrm{b}}, \mathrm{R}_{\mathrm{p}}, \alpha_{b}, \alpha_{p}$, where $\mathrm{R}_{\mathrm{b}}$ is the base radius, $\mathrm{R}_{\mathrm{p}}$ is the moving platform radius, angle $\alpha_{b}$ is from $\mathrm{X}$ axis to line $\mathrm{OB}_{1}$, angle $\alpha_{p}$ is from $\mathrm{x}$ axis to line $\mathrm{OP}_{1}$. The design variables' constraints are set as follows: $\mathrm{R}_{\mathrm{b}} \in[15,20] \mathrm{cm}, \mathrm{Rp} \in[6,12] \mathrm{cm}, \alpha_{b} \in$ $[0.35,0.7] \mathrm{rad}, \alpha_{p} \in[0,0.35] \mathrm{rad}$.

The differential evolution algorithm is employed here for optimizing the above objective function. The reason that differential evolution was chosen over the particle swarm optimization algorithm is that the differential evolution algorithm performs better than particle swarm optimization with regard to the solution accuracy, robustness and time cost. Furthermore, differential evolution can be implemented without great effort as compared to the other algorithms, and also differential evolution is much more efficient and robust in terms of convergent ability and producing the same end results in multiple runs as compared to other evolution algorithms. Storn and Price put forward the differential evolution in the year 1996. It is a stochastic and population-based optimization algorithm. Three main operations exist in differential evolution: mutation, crossover, and selection.

Mutation: In differential evolution algorithm, three vectors $\mathrm{Xa}, \mathrm{G}, \mathrm{X}_{\mathrm{b}, \mathrm{G}}$ and $\mathrm{Xc}, \mathrm{G}$ are chosen arbitrarily from the current population in order to perform the operation mutation.

$$
V \mathrm{i}, \mathrm{G}=X \mathrm{a},{ }_{\mathrm{G}}+\mathrm{F}\left(X_{\mathrm{b}}, \mathrm{G}^{-} X \mathrm{c}, \mathrm{G}_{\mathrm{G}}\right)
$$

Usually, $\mathrm{F}$ is a constant value ranging from zero to two and it determines the amplification of $\left(\mathrm{X}_{\mathrm{b}, \mathrm{G}}-\mathrm{Xc}, \mathrm{G}\right)$. Larger $\mathrm{F}$ value results in better diversity while lower value leads to faster convergence.

Crossover: New results can be produced through shuffling competing vectors and it is also possible to expand the population's diversity. The trial vector is defined as follows [28]:

$$
\begin{gathered}
U_{i, G}=\left(U_{1 i, G}, U_{2 i, G}, U_{3 i, G}, \ldots, U_{D i, G}\right) \\
U_{j i, G}= \begin{cases}V_{j i, G} & \text { if }(\text { rand } \leq C r) o r\left(j=j_{\text {rand }}\right) \\
X_{j i, G} & \text { otherwise }\end{cases}
\end{gathered}
$$

where $\mathrm{D}$ is the problem's dimension, $j_{\text {rand }}$ is a randomly selected factor to make sure that at least one parameter can be always chosen from $V_{j i, G} . C r \in(0,1)$ represents the crossover rate. rand represents a random value ranging from 0 to 1 .

Selection: The vector with better objective function value will be chosen and entered to the following iteration from the trial vector. For instance, for a minimum optimization issue, the vector that has the smallest objective function value will be chosen.

$$
X_{i}^{\prime}= \begin{cases}U_{i} & \text { if }\left(\operatorname{fitness}\left(U_{i}\right) \leq \text { fitness }\left(X_{i}\right)\right) \\ X_{i} & \text { otherwise }\end{cases}
$$

For the problem in this study, the below results can be obtained after optimization through employing the differential evolution algorithm:

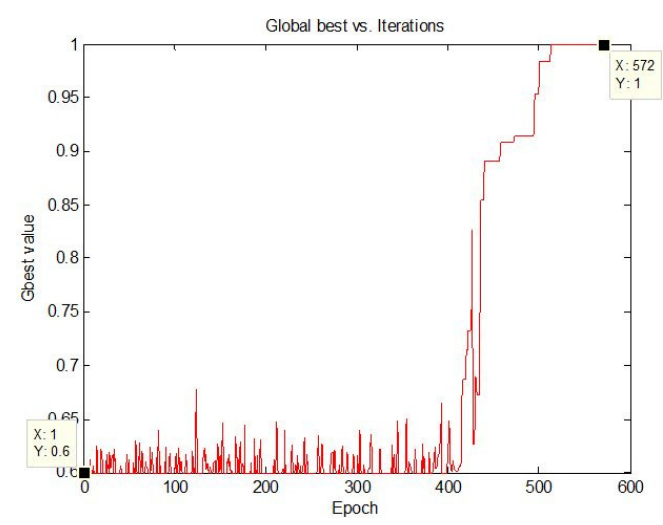

a) First trial

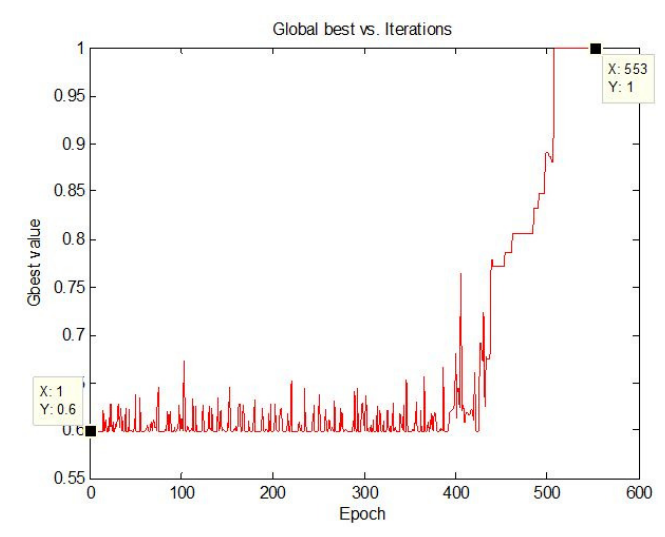

b) Second trial

Fig.4. Optimization results of stiffness employing differential evolution. 
The objective function's value converges to one after approximately 500 iterations. The performance graph illustrated in Fig.4. vibrates because $O b j F i_{\text {max }}$ and $O b j F i_{\text {min }}$ change in every single iteration. The $\mathrm{x}$ axis represents the number of iterations and the $y$ axis represents the best value discovered for the objective function in every single iteration. After performing the optimization, the base radius $\mathrm{R}_{\mathrm{b}}=20 \mathrm{~cm}$, the moving platform radius $\mathrm{R}_{\mathrm{p}}=6 \mathrm{~cm}$, the angle $\alpha_{b}$ from $\mathrm{X}$ axis to $\mathrm{OB}_{1}$ is $0.6981 \mathrm{rad}$, and the angle $\alpha_{p}$ from $\mathrm{x}$ axis to $\mathrm{OP}_{1}$ is zero. The summation of the $\mathrm{x}$ and $\mathrm{y}$ translational stiffness $O b j F 1=2729.4$, and the $\mathrm{Z}$ translational stiffness $O b j F 2=270.6122$.

\section{WORKSPACE OF THE MECHANISM AND SINGLE-OBJECTIVE OPTIMIZATION}

Regarding the optimization for the workspace, as mentioned earlier, the robotic mechanisms' workspace volume is usually employed to be an objective function to perform the optimization. One is able to calculate the volume through employing the fast search approach [21]. Under the case $\alpha_{b}=0.5 \mathrm{rad}, \alpha_{p}=0.25 \mathrm{rad}$, the volume changing trend with respect to $\mathrm{R}_{b}$ and $\mathrm{R}_{\mathrm{p}}$ is shown in Fig.5.

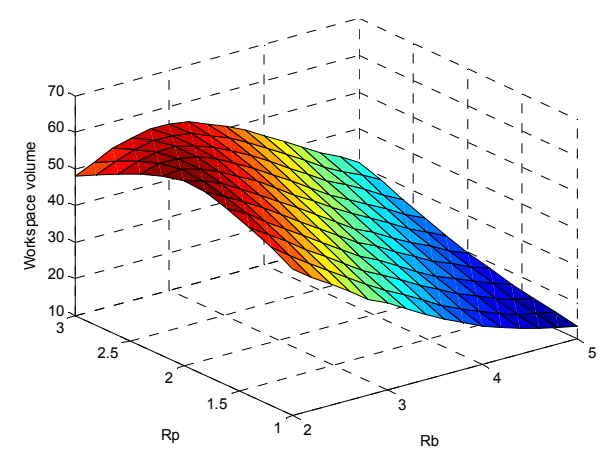

Fig.5. Workspace distribution along $R_{b}$ and $R_{p}$ when $\alpha_{b}=30^{\circ}, \alpha_{p}=15^{\circ}$.

When $\mathrm{R}_{\mathrm{b}}=4 \mathrm{~cm}, \mathrm{R}_{\mathrm{p}}=2 \mathrm{~cm}$, the volume changing trend with respect to $\alpha_{b}$ and $\alpha_{p}$ is shown in Fig.6.

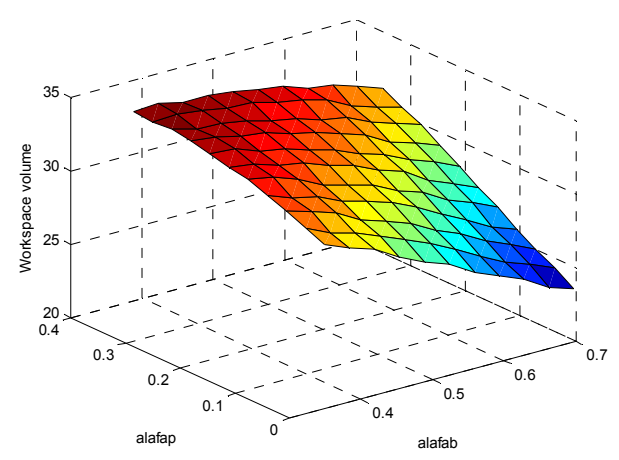

Fig.6. Workspace distribution with respect to $\alpha_{b}$ and $\alpha_{p}$ when $R_{b}=4, R_{p}=2$.

From Fig.5. and Fig.6., it is observed that the base radius, moving platform radius and angles on the two platforms all make a dent on the robotic mechanism's workspace volume. In the scenario where these four parameters alter at the same time, it will be very difficult to determine which parameters should be selected to produce the maximum volume of the workspace. Now the single-objective optimization is conducted through employing the differential evolution via adjusting the four design variables to generate the maximum volume for the workspace. The variables and its constraints are listed below: $\mathrm{R}_{\mathrm{b}} \in[2,5] \mathrm{cm}, \mathrm{R}_{\mathrm{p}} \in[1,3] \mathrm{cm}, \alpha_{b} \in$ $[0.35,0.7] \mathrm{rad}, \alpha_{p} \in[0,0.35] \mathrm{rad}$. After performing the optimization, the results shown below are generated:

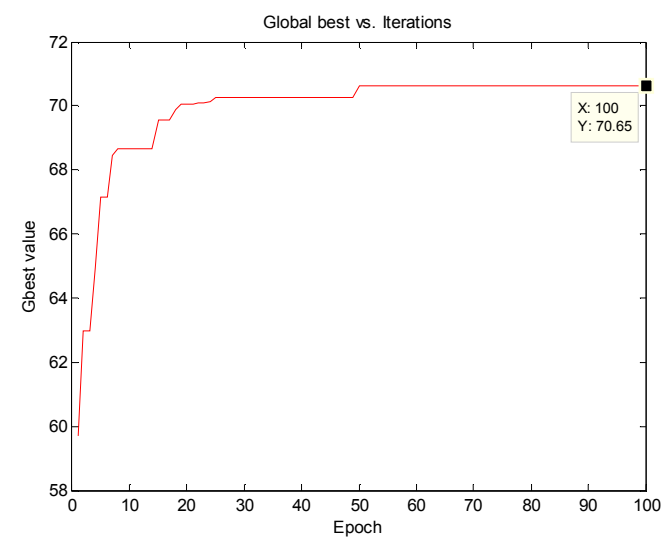

a) First time trial

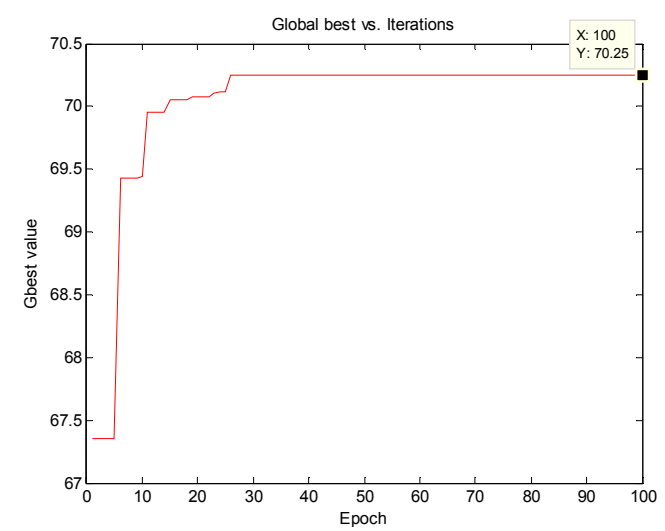

b) Second time trial

Fig.7. Optimization results of workspace volume employing DE.

From Fig.7., it is observed that the objective function is converging to 70.25 after approximately 50 iterations and the design variables are determined as $\mathrm{R}_{\mathrm{b}}=2.1163 \mathrm{~cm}$, $\mathrm{R}_{\mathrm{p}}=2.1244 \mathrm{~cm}, \quad \alpha_{b}=0.36 \mathrm{rad}, \quad \alpha_{p}=0.35 \mathrm{rad} \quad$ after optimization. Prior to performing the optimization, the volume of the workspace is 49.8384 . Fig.8. illustrates the comparison among the workspaces, one is after optimization case and another one is before optimization case. One can see that the volume of the workspace turns larger as compared to the one before optimization, and it is improved about 1.4 times. 


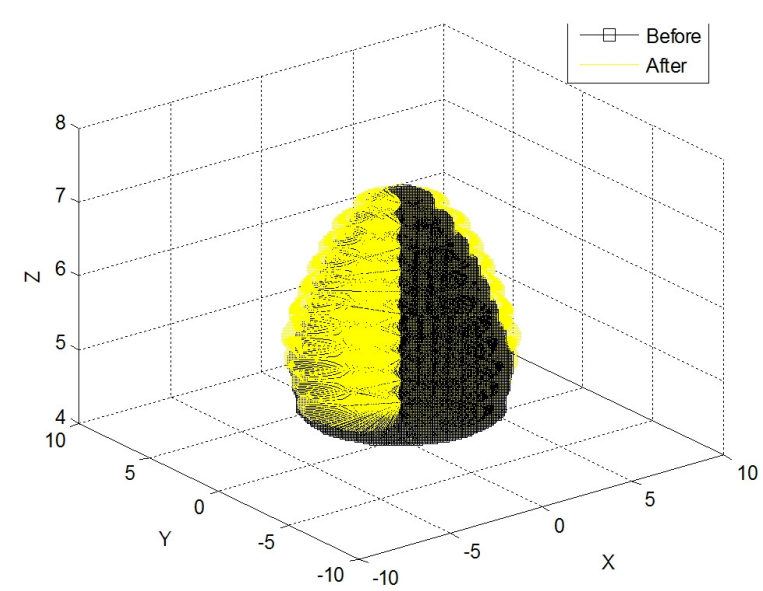

Fig.8. Workspace comparison after optimization and prior to optimization.

\section{MULTI-OBJECTIVE OPTIMIZATION FOR STIFFNESS AND WORKSPACE}

Usually in most scenarios for the parallel mechanisms, when the stiffness increases, the workspace is influenced by that. Here, the changing trends for the stiffness and workspace with respect to $\mathrm{R}_{\mathrm{b}}, \mathrm{R}_{\mathrm{p}}, \alpha_{b}$ and $\alpha_{p}$ are firstly investigated in order to see if these two terms conflict or not. In the case where $\alpha_{b}=0.5 \mathrm{rad}, \alpha_{p}=0.25 \mathrm{rad}$, the changing trends for the summation of the $\mathrm{x}$ and $\mathrm{y}$ directional stiffness, $\mathrm{z}$ directional stiffness and the volume of the workspace with respect to $R_{b}$ and $R_{p}$ are illustrated in Fig.9.

When $R_{b}=4 \mathrm{~cm}, R_{p}=2 \mathrm{~cm}$, the changing trends for the summation of the $\mathrm{x}$ and $\mathrm{y}$ translational stiffness and the volume of the workspace with respect to $\alpha_{b}$ and $\alpha_{p}$ are demonstrated in Fig. 10.

From Fig.9. and Fig.10,, one can see that the $z$ translational stiffness and the volume of the workspace possess the same changing trend while the summation of $\mathrm{x}$ and $y$ translational stiffness and the volume possess the reversing changing trend. In the case where the $z$ translational stiffness increases, the volume of the workspace increases as well, this is what is desired. However, in the case where the $\mathrm{x}$ and $\mathrm{y}$ translational stiffness increase, the volume decreases, which is not what is wanted, these two conflict with each other. Thus, one can employ the differential evolution to single-objective optimize the $\mathrm{z}$ translational stiffness independently without taking the performance of the workspace into consideration. However, the $\mathrm{z}$ translational stiffness has the reverse changing trend with the $\mathrm{x}$ and $\mathrm{y}$ translational stiffness, when one resorts to the differential evolution to optimize the $\mathrm{z}$ translational stiffness, the $\mathrm{x}$ and $\mathrm{y}$ translational stiffness will get influenced. Thus, in practical applications, engineers need to take into consideration what factor is more important to them in order to select one to perform the optimization, i.e. $\mathrm{z}$ translational stiffness or $\mathrm{x}$ and $\mathrm{y}$ translational stiffness. As mentioned earlier, the reason the differential evolution is preferably selected here is that it is more efficient and robust in terms of convergent ability and produces the same results in multiple trails as compared to other evolutionary algorithms. Additionally, the differential evolution algorithm generally performs better than the particle swarm optimization with respect to solution accuracy, robustness and time cost. Furthermore, the differential evolution is easier to be implemented as compared to the other algorithms [25]. The authors here deem that the $\mathrm{x}$ and $\mathrm{y}$ translational stiffness is more critical than $\mathrm{z}$ translational stiffness. The summation of the $\mathrm{x}$ and $\mathrm{y}$ translational stiffness and the volume of the workspace are being optimized at the same time through employing the Pareto front theory.
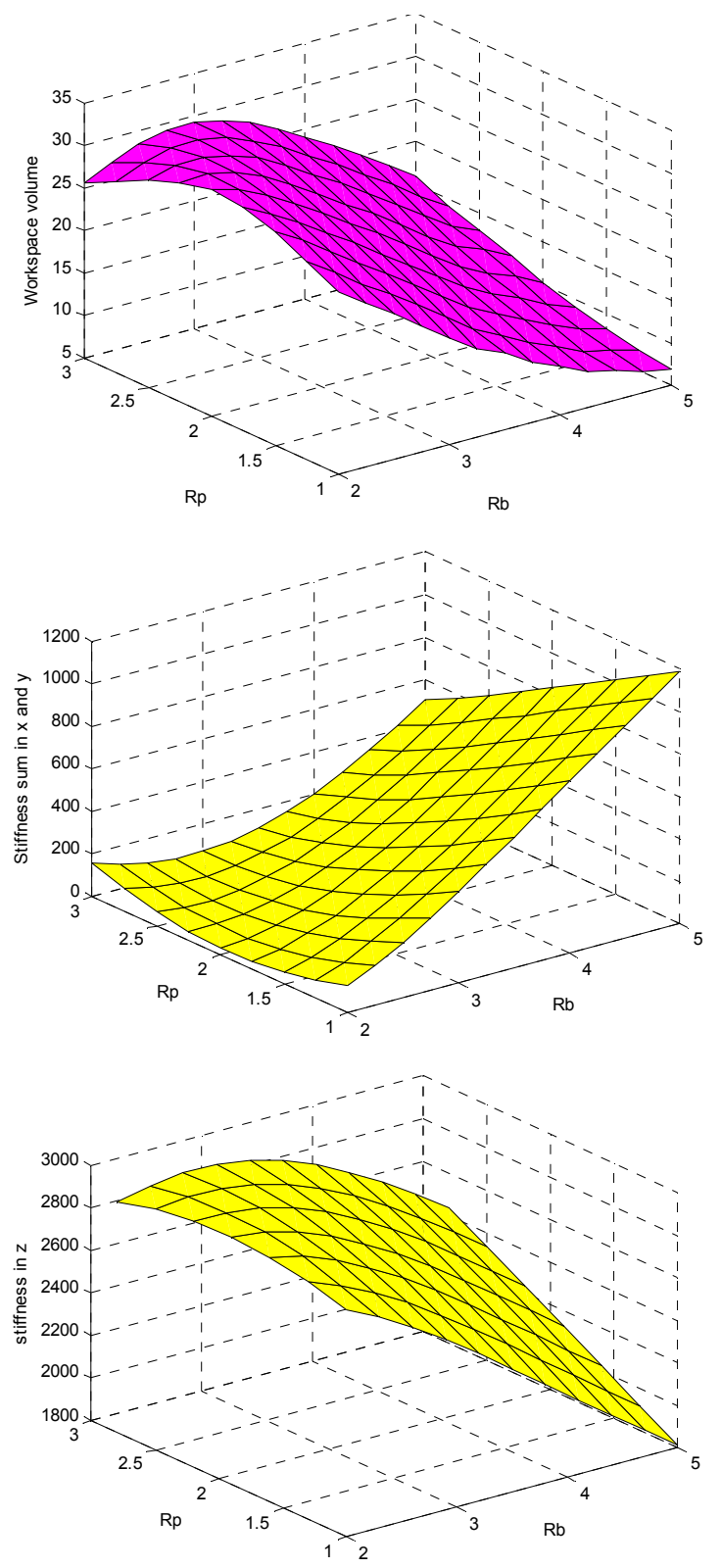

Fig.9. Summation of $\mathrm{x}$ and $\mathrm{y}$ directional stiffness, $\mathrm{z}$ directional stiffness and volume of workspace along $R_{b}$ and $R_{p}$ when $\alpha_{b}=30^{\circ}, \alpha_{p}=15^{\circ}$. 

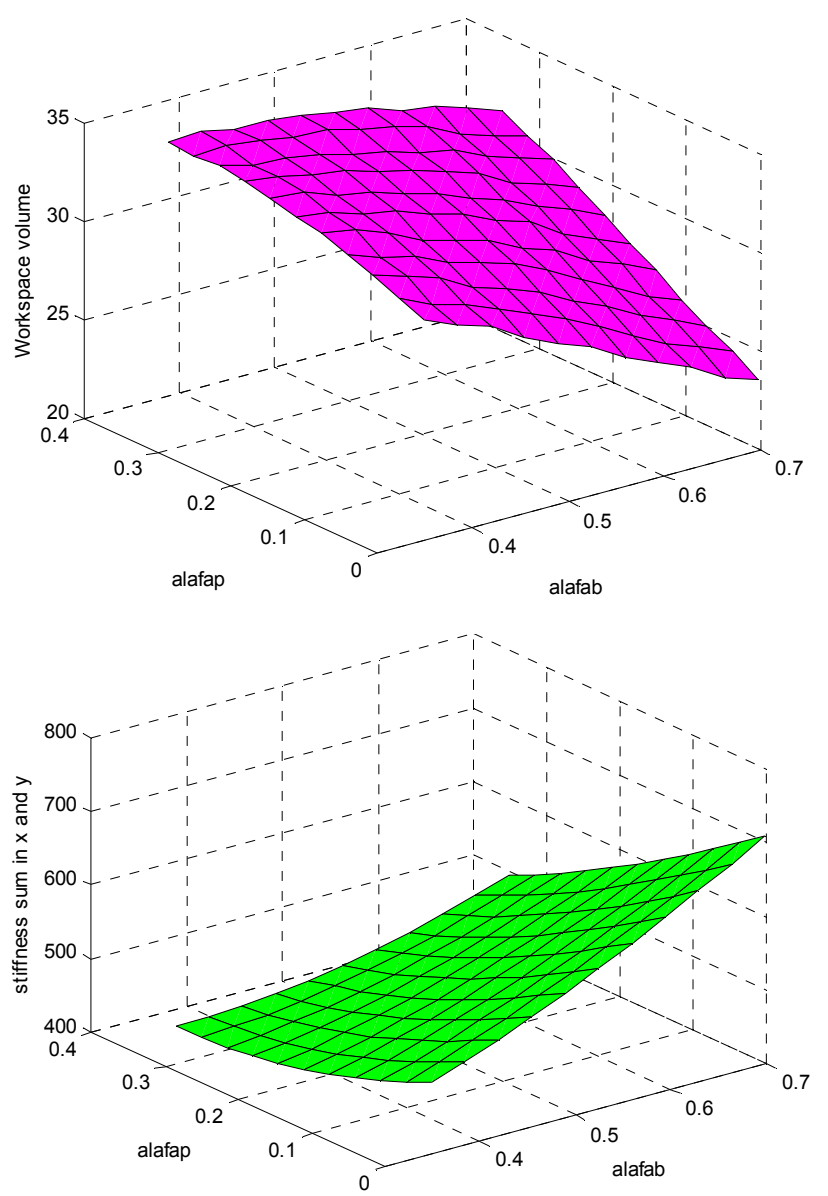

Fig.10. Summation of $\mathrm{x}$ and $\mathrm{y}$ directional stiffness and the volume of the workspace along with $\alpha_{b}$ and $\alpha_{p}$ when $R_{b}=4, R_{p}=2$.

Objective function 1:

$$
\operatorname{Obj} F 1=K(1,1)+K(2,2)
$$

where ObjF1 represents the summation of the $\mathrm{x}$ and $\mathrm{y}$ directional stiffness. Maximizing ObjF1 is equivalent to minimize -ObjF1.

Objective function 2:

$$
\text { ObjF } 2=\text { WorkspaceVolume }
$$

where $\mathrm{ObjF} 2$ represents the volume of the workspace for the robotic mechanism. Similarly, maximizing $\mathrm{ObjF} 2$ is equivalent to minimizing -ObjF2.

Design variables are $\mathrm{R}_{\mathrm{b}}, \mathrm{R}_{\mathrm{p}}, \alpha_{b}$ and $\alpha_{p}$. The constraints are listed below: $\mathrm{R}_{\mathrm{b}} \in[2,5] \mathrm{cm}, \mathrm{R}_{\mathrm{p}} \in[1,3] \mathrm{cm}, \alpha_{b} \in$ $[0.35,0.7] \mathrm{rad}$ and $\alpha_{p} \in[0,0.35] \mathrm{rad}$. The authors chose the population size to be 50 , the maximum of generations to be 100 , the selection strategy as tournament, the tournament size to be 2 , the crossover type as intermediate, the crossover ratio to be 1 , the mutation function to be as adaptive feasible, and the Pareto front population fraction to be 0.35 . After performing the optimization, the Pareto front of the objective functions 1 (summation of $\mathrm{x}$ and $\mathrm{y}$ translational stiffness) and 2 (volume of workspace) is generated (illustrated in Fig.11.). It can be observed that the summation of the $\mathrm{x}$ and $\mathrm{y}$ translational stiffness and the volume of the workspace do conflict with one another. When the summation of the $\mathrm{x}$ and $\mathrm{y}$ translational stiffness is increasing, the volume of the workspace is decreasing and vice versa.

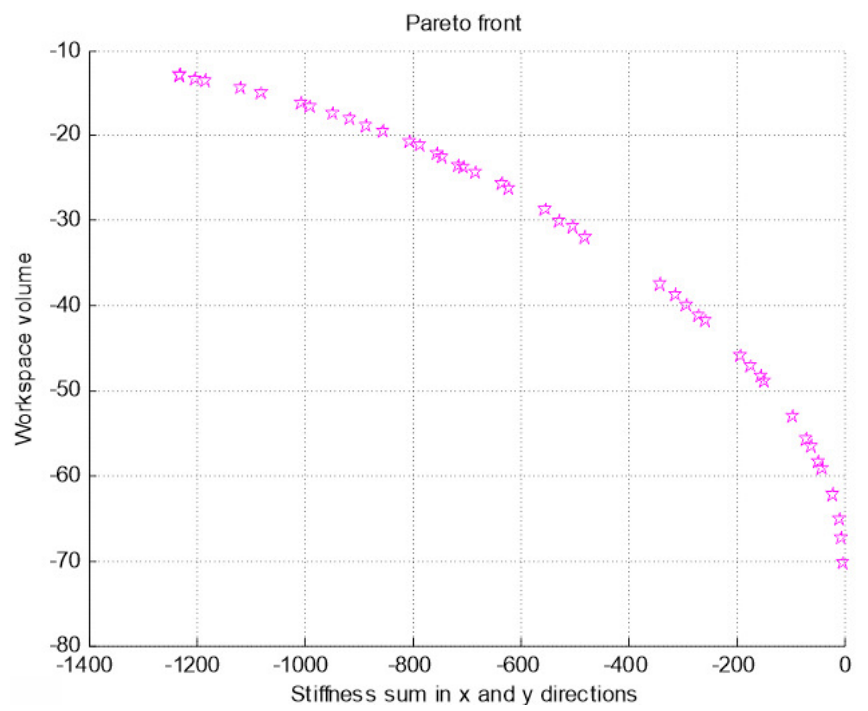

Fig.11. Pareto front sets between stiffness and workspace.

There is not one optimal solution as it is the case for the single-objective case, but multiple solutions, to which they are non-dominate. Some of the objective values and values for the design variables are recorded in Table 1. Here, the authors will not enumerate all of them as there are too many. Engineers are able to select specific ones that are based on their own requirements. In the scenario where engineers demand larger stiffness and the volume of the workspace is not vital, the one that has larger value for the stiffness can be selected and some workspace can be compromised to some extent. In the scenario where engineers deem workspace critical, they can select a larger value for workspace and compromise some stiffness.

Table 1. Pareto front solutions.

\begin{tabular}{|l|l|l|l|l|l|}
\hline ObjF1 & ObjF2 & $R_{b}$ & $R_{p}$ & $\alpha_{b}$ & $\alpha_{p}$ \\
\hline-4.0869 & -67.3870 & 2.1198 & 2.0068 & 0.3950 & 0.3242 \\
\hline-1004.638 & -16.2115 & 4.7256 & 1.3232 & 0.6025 & 0.1977 \\
\hline-269.7363 & -41.1384 & 2.8959 & 1.3991 & 0.5062 & 0.2676 \\
\hline
\end{tabular}

The Pareto front theory was employed to multi-objective optimize the summation of the $\mathrm{x}$ and $\mathrm{y}$ translational stiffness and the volume. Here the authors are also able to transform the multi-objective optimization issue into a single-objective case via the formulation below:

$$
O b j F=O b j F 1 \cdot O b j F 2
$$


Through Equation (10), the multi-objective case becomes a single-objective case. The design variables are $\mathrm{R}_{\mathrm{b}}, \mathrm{R}_{\mathrm{p}}, \alpha_{b}$ and $\alpha_{p}$, and the constraints are listed below: $\mathrm{R}_{\mathrm{b}} \in[2,5] \mathrm{cm}, \mathrm{R}_{\mathrm{p}} \in[1,3] \mathrm{cm}, \alpha_{b} \in[0.35,0.7] \mathrm{rad}$, $\alpha_{p} \in[0,0.35] \mathrm{rad}$. After performing the optimization, the following are generated:

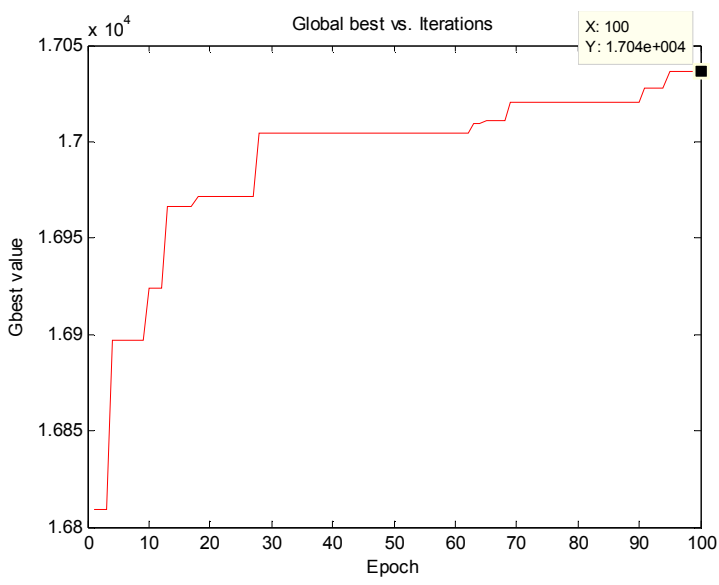

Fig.12. Optimization results employing DE.

It can be seen that the objective function is converging to $1.704 \times 10^{4}$ after about 100 generations, and the values for the design variables are $R_{b}=4.2876 \mathrm{~cm}, R_{p}=1.7948 \mathrm{~cm}$, $\alpha_{b}=0.6267 \mathrm{rad}$ and $\alpha_{p}=0.0629 \mathrm{rad}$ after the optimization. Prior to the optimization, $R_{b}=3 \mathrm{~cm}$, $R_{p}=2 \mathrm{~cm}, \quad \alpha_{b}=0.523 \mathrm{rad}, \quad \alpha_{p}=0.3 \mathrm{rad} \quad$ and $O b j F=6.7516 \times 10^{3} . O b j F$ has improved approximately 2.5 times after the optimization. It is observed that the single-objective optimization possesses only one optimal solution while the multi-objective case possesses multiple solutions, from which engineers are able to select based on their specific requirements and preferences, thus the final selection is depending on engineers.

\section{PoteNTIAL APPLICATION - PARALLEL ROBOTIC BASED ASSISTED MACHINE FOR STANDING AND WALKING EXERCISE FOR STROKE PATIENTS}

\section{A. Background}

Nowadays, unfortunately many people have suffered from stroke, which causes people to have lasting consequences, i.e. people who suffer from stroke have physical disabilities or even worse (become vegetable person). Our study focuses on how to help those patients exercise so that patients with disabilities can be prevented from organ deterioration and muscle shrinks. In this section, the authors propose an assisted exercise standing and walking machine based on the parallel robotic structure for those patients. To the best of the authors' knowledge, this will be the first exercise machine for patients with stroke disability based on the parallel robotic structure.
When people suffer from stroke, sometimes the patients will not have any lasting consequences and act like a normal person, but sometimes people will have severe disabilities, the symptom can be that patients lose walking and standing abilities, or sometimes patients become vegetable persons. For the latter case, if the patients are bed ridden for a long time, their organs will deteriorate and their muscles will shrink. In most cases, in second world countries, such as in China and India, etc., where a large number of stroke patients reside, two or three family members just hold the patients and help the patients stand and walk, which is extremely energy consuming for the helpers.

\section{B. Solution - Parallel robotic based exercise machine}

To overcome the above problem, the authors designed an exercise standing and walking machine for the patients with stroke disabilities based on the parallel robotic structure, as shown in Fig.1. The machine can not only help patients stand, but also can help patients walk, so as to act as an exercise machine in order to prevent patients from organ deterioration and muscle shrink. The machine consists of two main parts, the lower component and upper component. The lower component mainly consists of three 3-UPU parallel robotic mechanisms, $U$ stands for the universal joint, P stands for the prismatic joint. Each 3-UPU parallel robotic mechanism has three translational degrees of freedom. These translational degrees of freedom enable the patients' legs to move up and down and move forward and backward. The upper component can prevent the patients' upper body from falling, and the lower component makes the patients move around.

The machine consists of the following components: part 1 is the moving platform used to support the neck section of a human body, the middle platform 3 is connected to the moving platform 1 with several links, and they are denoted as part 2. The middle platform is used to support the waist of a human body. The middle platform is connected to the base platform (part 11) through universal joints (part 4), actuated prismatic joints (part 6) and links (parts 5 and 7). There are two wheels (part 8) in the base platform, which is used to move the whole machine when a patient finishes a single step cycle. Part 9 is the opening section that allows patients to get into this machine. Part 10 is a motor that is used to actuate the prismatic joints. There are another two 3-UPU mechanisms in the middle, which are used to move the legs of the patients, i.e. lift up the legs of the patients, move forward the legs and put down the legs on the floor. In the meantime, the large 3-UPU mechanism is used to move the waist section of a human body to move along with the legs. When the patients wear this machine, we only need to slightly move the machine when the patients finish a step cycle, which can greatly alleviate our helpers. The proposed parallel robotic based assisted machine will be prototyped according to the optimized results in the previous section and it will be further tested by the patients in terms of suitability of the proposed machine in the near future. 


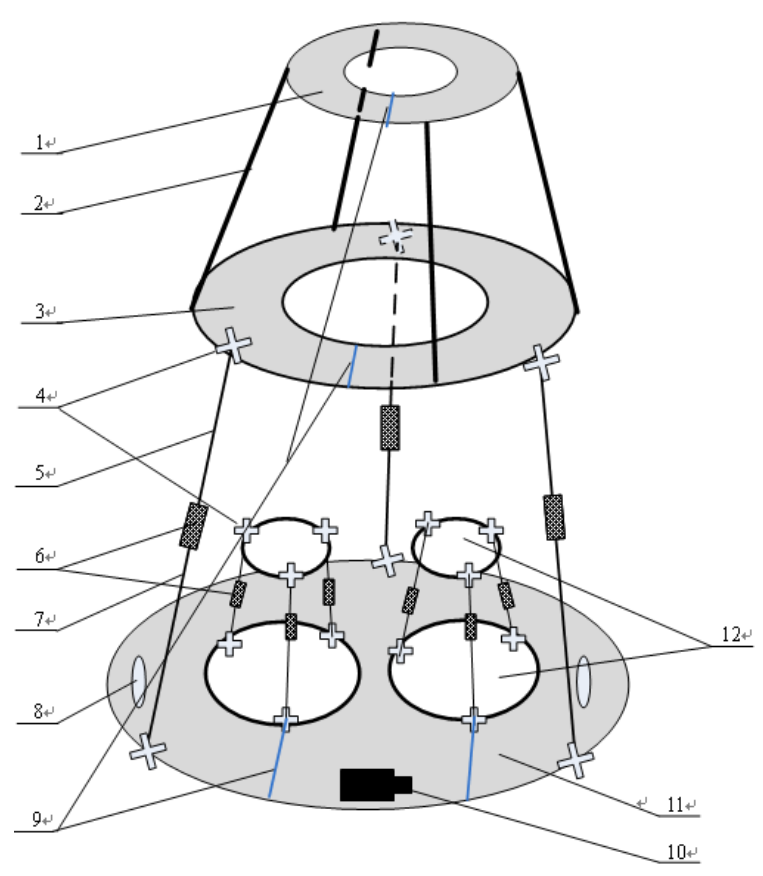

Fig.13. Parallel robotic based assisted machine.

\section{CONCLUSION}

The interactions between stiffness and workspace performances are investigated in this paper, and also the stiffness and workspace of a 3-UPU robotic mechanism are optimized, respectively. Since the summation of the $\mathrm{x}$ and $\mathrm{y}$ directional stiffness and the $\mathrm{z}$ directional stiffness are not in the same scale, a normalization of the two objectives is performed. Furthermore, via analyzing it was observed that the changing trends for the $\mathrm{x}$ and $\mathrm{y}$ translational stiffness and the volume of the workspace are reversing whereas the $\mathrm{z}$ translational stiffness and the volume of the workspace possess the same changing trends. One can single-objective optimize the $\mathrm{z}$ translational stiffness independently without taking into consideration the volume of the workspace. However, the $\mathrm{z}$ translational stiffness shows a reversing changing trend with respect to the $\mathrm{x}$ and $\mathrm{y}$ directional stiffness. Thus, in the case where one optimizes the $\mathrm{z}$ translational stiffness, the $\mathrm{x}$ and $\mathrm{y}$ directional stiffness get affected. In practical applications, engineers need to determine which factor is more critical to them in order to select one factor to perform the optimization. As an example, through employing the Pareto front theory and also via employing the differential evolution algorithm by transforming the multi-objective optimization issue to the single-objective optimization issue, respectively, the summation of the $\mathrm{x}$ and $\mathrm{y}$ translation and the volume of the workspace for the robotic mechanism are being optimized at the same time. The results indicate the kinematic performances are able to be improved after performing the optimization. Furthermore, it is observed that the multiobjective optimization possesses multiple solutions in the Pareto front, to which they are non-dominate, and engineers are able to select one solution based on their specific requirements and preferences, while the single-objective optimization possesses only one optimal solution. The mechanism can be potentially used in integrating the exercise-walking machine for stroke patients. As for the future work, the global stiffness will be considered. Due to the space limitation, some comparison studies will be conducted in the next step. In addition, the proposed parallel robotic based assisted machine will be prototyped according to the optimized results and the patients will try it out in order to further test the suitability of the proposed machine.

\section{ACKNOWLEDGMENT}

The authors would like to thank the financial support from the Natural Sciences and Engineering Research Council of Canada (NSERC) and the Canada Research Chairs (CRC) program.

\section{REFERENCES}

[1] Bohez, E. (2002). Five-axis milling machine tool kinematic chain design and analysis. International Journal of Machine Tools \& Manufacture, 42, 505520.

[2] Tsai, L., Joshi, S. (2002). Kinematic analysis of 3DOF position mechanisms for use in hybrid kinematic machines. Journal of Mechanical Design, 124 (2), 245-253.

[3] Zhang, D., Bi, Z., Li, B. (2009). Design and kinetostatic analysis of a new parallel manipulator. Robotics and Computer-Integrated Manufacturing, 25 (4-5), 782-791.

[4] Castelli, G., Ottaviano, E. (2009). Modeling and simulation of a cable based parallel manipulator as an assisting device. In Computational Kinematics: Proceedings of the 5th International Workshop on Computational Kinematics. Springer, 17-24.

[5] Li, Y., Xu, Q. (2005). Kinematic design and dynamic analysis of a medical parallel manipulator for chest compression task. In IEEE International Conference on Robotics and Biomimetics (ROBIO), July 5-9, 2005. IEEE, 693-698.

[6] Plitea, N., Pisla, D., Vaida, C. (2007). On kinematics of a parallel robot used for the minimally invasive surgery. PAMM - Proceedings in Applied Mathematics and Mechanics, 7 (1), 4010033-4010034.

[7] Lessard, S., Bigras, P., Bonev, I. (2007). A new medical parallel robot and its static balancing optimization. Journal of Medical Devices, 1, 272-278.

[8] Liang, Q., Wu, W., Zhang, D., Wei, B., Sun, W., Wang, Y., Ge, Y. (2015). Design and analysis of a micromechanical three-component force sensor for characterizing and quantifying surface roughness. Measurement Science Review, 15 (5), 248-255.

[9] Merlet, J.P. (2006). Parallel Robots. Springer.

[10] Zhang, D. (2010). Parallel Robotic Machine Tools. Springer.

[11] Bruckmann, T., Mikelsons, L., Brandt, T., Hiller, M., Schramm, D. (2008). Wire robots Part I.: Kinematics, analysis and design. In Parallel Manipulators: New Developments. I-Tech Education and Publishing, 109132. 
[12] Gosselin, C. (1990). Stiffness mapping for parallel manipulators. IEEE Transactions on Robotics and Automation, 6 (3), 377-382.

[13] Carbone, G. (2011). Stiffness analysis and experimental validation of robotic systems. Frontiers of Mechanical Engineering, 6 (2), 182-196.

[14] Zhang, D., Gao, Z. (2012). Forward kinematics, performance analysis, and multi-objective optimization of a bio-inspired parallel manipulator. Robotics and Computer-Integrated Manufacturing, 28 (4), 484-492.

[15] Zhang, D., Xi, F., Mechefske, C., Lang, S. (2004). Analysis of parallel kinematic machine with kinetostatic modelling method. Robotics and Computer-Integrated Manufacturing, 20 (2), 151-165.

[16] Li, J. (2009). Design of 3-DOF parallel manipulators for micromotion applications. Master Thesis, University of Ontario Institute of Technology.

[17] Li, B., Wang, Z., Hu, Y. (1999). The stiffness calculation model of the new typed parallel machine tool. Machine Design, 3, 14-16.

[18] Gosselin, C., Angeles, J. (1991). A global performance index for the kinematic optimization of robotic manipulators. Journal of Mechanical Design, 113 (3), 220-226.

[19] Stamper, R., Tsai, L., Walsh, G. (1997). Optimization of a three DOF translational platform for wellconditioned workspace. In IEEE International Conference on Robotics and Automation, April 25, 1997. IEEE, 3250-3255.

[20] Gao, Z., Zhang, D. (2011). Workspace representation and optimization of a novel parallel mechanism with three-degrees-of-freedom. Sustainability, 3, 22172228 .
[21] Masory, O., Wang, J. (1995). Workspace evaluation of Stewart platforms. Advanced Robotics, 9 (4), 443-461.

[22] Zhang, D., Gao, Z. (2012). Multi-objective performance optimization of a parallel robotic machine tool. In IEEE/ASME International Conference on Mechatronics and Embedded Systems and Applications (MESA), July 8-10, 2012. IEEE, 154-159.

[23] Coppola, G., Zhang, D., Liu, K.F. (2014). A 6-DOF reconfigurable hybrid parallel manipulator. Robotics and Computer-Integrated Manufacturing, 30 (2), 99106.

[24] Chi, Z., Zhang, D., Xia, L., Gao, Z. (2013). Multiobjective optimization of stiffness and workspace for a parallel kinematic machine. International Journal of Mechanics and Materials in Design, 9, 281-293.

[25] Vesterstrom, J., Thomsen, R. (2004). A comparative study of differential evolution, particle swarm optimization, and evolutionary algorithms on numerical benchmark problems. In Congress on Evolutionary Computation (CEC2004), June 19-23, 2004. IEEE, vol. 2, 1980-1987.

[26] Zhang, D. (2000). Kinetostatic analysis and optimization of parallel and hybrid architectures for machine tools. Ph.D. thesis, Laval University, Quebec, Canada.

[27] Talbi, E.G. (2009). Metaheuristics: From Design to Implementation. John Wiley \& Sons.

[28] Rahnamayan, S. (2007). Opposition-based differential evolution. Ph.D. thesis, University of Waterloo, Ontario, Canada. 